\title{
Atomic decompositions, two stars theorems, and distances for the Bourgain- Brezis-Mironescu space and other big spaces
}

Article

Accepted Version

Creative Commons: Attribution-Noncommercial-No Derivative Works 4.0

D'Onofrio, L., Greco, L., Perfekt, K.-M., Sbordone, C. and Schiattarella, R. (2020) Atomic decompositions, two stars theorems, and distances for the Bourgain-Brezis-Mironescu space and other big spaces. Annales de l'Institut Henri Poincare (C) Analyse Non Lineaire, 37 (3). pp. 653-661. ISSN 0294-1449 doi: https://doi.org/10.1016/j.anihpc.2020.01.004 Available at https://centaur.reading.ac.uk/88445/

It is advisable to refer to the publisher's version if you intend to cite from the work. See Guidance on citing.

To link to this article DOI: http://dx.doi.org/10.1016/j.anihpc.2020.01.004

Publisher: Elsevier

All outputs in CentAUR are protected by Intellectual Property Rights law, including copyright law. Copyright and IPR is retained by the creators or other copyright holders. Terms and conditions for use of this material are defined in the End User Agreement. 


\section{www.reading.ac.uk/centaur}

\section{CentAUR}

Central Archive at the University of Reading

Reading's research outputs online 


\title{
ATOMIC DECOMPOSITIONS, TWO STARS THEOREMS, AND DISTANCES FOR THE BOURGAIN-BREZIS-MIRONESCU SPACE AND OTHER BIG SPACES
}

\author{
LUIGI D'ONOFRIO, LUIGI GRECO, KARL-MIKAEL PERFEKT, CARLO SBORDONE, \\ AND ROBERTA SCHIATTARELLA
}

\begin{abstract}
Given a Banach space $E$ with a supremum-type norm induced by a collection of operators, we prove that $E$ is a dual space and provide an atomic decomposition of its predual. We apply this result, and some results obtained previously by one of the authors, to the function space $\mathcal{B}$ introduced recently by Bourgain, Brezis, and Mironescu. This yields an atomic decomposition of the predual $\mathcal{B}_{*}$, the biduality result that $\mathcal{B}_{0}^{*}=\mathcal{B}_{*}$ and $\mathcal{B}_{*}^{*}=\mathcal{B}$, and a formula for the distance from an element $f \in \mathcal{B}$ to $\mathcal{B}_{0}$.
\end{abstract}

\section{INTRODUCTION}

Suppose that a Banach space $E$ is defined and normed by the fact that $x \in E$ if and only if $\sup _{L \in \mathcal{L}}\|L x\|_{Y}<\infty$, where $\mathcal{L}$ is a collection of operators $L: X \rightarrow Y, X$ and $Y$ Banach spaces. Spaces of this kind include the space of bounded mean oscillation (BMO), Hölder spaces, the space of bounded variation (BV), Marcinkiewicz spaces, and various spaces of holomorphic functions of weighted or Möbius invariant type.

Most of these spaces are known to have a predual $E_{*}$ whose elements can be defined in terms of a decomposition into designated "atoms". See for instance $[4,6]$ for two recent examples related to what will be our main application. While the general line of argument to obtain such atomic decompositions has appeared frequently and repeatedly, the main purpose of this note is to provide a completely functional analytic proof, independent of any special structure of $\mathcal{L}$. In particular, our approach can be used to recover some of the results from $[4,6]$, as well as many other examples of atomic decompositions in the literature.

Another typical result, in the cases where it is possible to define a sufficiently rich "vanishing" subspace $E_{0} \subset E$, is the "two stars theorem". Namely, that $E_{0}^{*}=E_{*}$. Furthermore, the distance from an element $f \in E$ to $E_{0}$ is usually given by an appropriate limit of the defining functionals. The pair $(\mathrm{BMO}(\mathbb{T}), \mathrm{VMO}(\mathbb{T}))$ provides a familiar example. These phenomena were formalized by one of the authors in [13, 14], and they were proven to hold in a fairly general context (without giving any description of $E_{*}$ ). See also [5] for a survey.

Our second purpose is to demonstrate the application of these results to the new function space $\mathcal{B}$ introduced by Bourgain, Brezis, and Mironescu [3]. Recent work related to this space can also be found in $[1,2,7,8,9]$. For $d \geq 1, f \in L^{1}\left((0,1)^{d}\right)$, and a cube $Q_{\varepsilon} \subset(0,1)^{d}$ with sides of length $\varepsilon$ and parallel to the co-ordinate axes, let $f_{Q_{\varepsilon}}$ denote the average of $f$ on $Q_{\varepsilon}$. Define the norm (modulo constants)

$$
\|f\|_{\mathcal{B}}:=\sup _{0<\varepsilon \leq 1}[f]_{\varepsilon}
$$

Date: December 18, 2019. 
where

$$
[f]_{\varepsilon}=\varepsilon^{d-1} \sup _{\mathcal{F}_{\varepsilon}} \sum_{Q_{\varepsilon} \in \mathcal{F}_{\varepsilon}} \frac{1}{\left|Q_{\varepsilon}\right|} \int_{Q_{\varepsilon}}\left|f(x)-f_{Q_{\varepsilon}}\right| d x .
$$

Here $\mathcal{F}_{\epsilon}$ denotes a collection of mutually disjoint $\varepsilon$-cubes $Q_{\varepsilon} \subset(0,1)^{d}$ such that the cardinality $\left|\mathcal{F}_{\varepsilon}\right| \leq \varepsilon^{1-d}$, and the supremum is taken over all such collections. The space $\mathcal{B}$ is then defined as

$$
\mathcal{B}=\left\{f \in L^{1}\left((0,1)^{d}\right):\|f\|_{\mathcal{B}}<\infty\right\} .
$$

When $d=1, \mathcal{B}=\mathrm{BMO}$. For $d \geq 2$, the $\mathcal{B}$-norm is strictly weaker than the BMO-norm. In fact, both BMO and BV are continuously contained in $\mathcal{B}$ (see [3]).

The separable vanishing subspace $\mathcal{B}_{0}$ consists of those $f \in \mathcal{B}$ such that

$$
\varlimsup_{\varepsilon \rightarrow 0}[f]_{\varepsilon}=0 .
$$

VMO and $W^{1,1}$ are continuously contained in $\mathcal{B}_{0}$.

For the space $\mathcal{B}$, our result yields the following.

Theorem 1. $\mathcal{B}$ has an (isometric) predual $\mathcal{B}_{*}$. Every $\varphi \in \mathcal{B}_{*}$ is of the form

$$
\varphi=\sum_{n=1}^{\infty} \lambda_{n} g_{n}
$$

where $\left(\lambda_{n}\right) \in \ell^{1}$ and each atom $g_{n}$ is associated with an $\varepsilon=\varepsilon_{n}$ and a collection of disjoint $\varepsilon$-cubes $\mathcal{F}_{\varepsilon}$ such that $\left|\mathcal{F}_{\varepsilon}\right| \leq \varepsilon^{1-d}$ and

- $\operatorname{supp} g_{n} \subset \cup \mathcal{F}_{\varepsilon}$,

- $\left|g_{n}\right| \chi_{Q_{\varepsilon}} \leq \varepsilon^{d-1} \frac{1}{\left|Q_{\varepsilon}\right|}$ for every $Q_{\varepsilon} \in \mathcal{F}_{\varepsilon}$,

- $\int_{Q_{\varepsilon}} g_{n} d x=0$ for every $Q_{\varepsilon} \in \mathcal{F}_{\varepsilon}$.

The action of $f \in \mathcal{B}$ on $\varphi$ is given by

$$
f(\varphi)=\sum_{n=1}^{\infty} \lambda_{n} \int_{(0,1)^{d}} f g_{n} d x
$$

and is independent of the choice of representation of $\varphi$. Furthermore,

$$
\frac{1}{2} \inf \sum_{n=1}^{\infty}\left|\lambda_{n}\right| \leq\|\varphi\|_{B_{*}} \leq \inf \sum_{n=1}^{\infty}\left|\lambda_{n}\right|,
$$

where the infimum is taken over all representations of $\varphi$.

Remark. As expected, the result shows that $\mathcal{B}_{*}$ is continuously contained in the atomic Hardy space $H^{1}\left((0,1)^{d}\right)$. In particular, the convergence of the series (2) can be understood in $H^{1}$, and thus in $L^{1}$.

As mentioned before, we will also show the following biduality and distance result. The meaning of the duality relations will be made more precise in Section 3 . Let UC $\subset \mathcal{B}_{0}$ denote the space of uniformly continuous functions on $(0,1)^{d}$ (modulo constants). Note that UC is dense in $\mathcal{B}_{0}$, by the remark after Lemma 7.

Theorem 2. We have that $\mathcal{B}_{0}^{*}=\mathcal{B}_{*}$ and $\mathcal{B}_{0}^{* *}=\mathcal{B}_{*}^{*}=\mathcal{B}$, isometrically via the $L^{2}\left((0,1)^{d}\right)$ pairing. For any $f \in \mathcal{B}$ it holds that

$$
\operatorname{dist}\left(f, \mathcal{B}_{0}\right)=\operatorname{dist}(f, \mathrm{UC})=\min _{g \in \mathcal{B}_{0}}\|f-g\|_{\mathcal{B}}=\varlimsup_{\varepsilon \rightarrow 0}[f]_{\varepsilon} .
$$

Remark. The result of [14] implies that $\mathcal{B}_{0}$ is an $M$-ideal in $\mathcal{B}$. Thus, by $M$-structure theory [11], the minimizer of (3) exists but is never unique, unless $f \in \mathcal{B}_{0}$. 
Remark. If the constraint on the cardinality, $\left|\mathcal{F}_{\varepsilon}\right| \leq \varepsilon^{1-d}$, is removed from the definition of $\mathcal{B}$, we find the familiar space $\mathrm{BV}$ of functions of bounded variation on $(0,1)^{d}$. Indeed, it can easily be shown that $f \in \mathrm{BV}$ if and only if

$$
\sup _{0<\varepsilon \leq 1} \varepsilon^{d-1} \sup _{\mathcal{G}_{\varepsilon}} \sum_{Q_{\varepsilon} \in \mathcal{G}_{\varepsilon}} \frac{1}{\left|Q_{\varepsilon}\right|} \int_{Q_{\varepsilon}}\left|f(x)-f_{Q_{\varepsilon}}\right| d x<\infty,
$$

where the supremum now runs over all families $\mathcal{G}_{\varepsilon}$ of pairwise disjoint $\varepsilon$-cubes contained in $(0,1)^{d}$. Actually, this is a particular case of [7, Corollary 4.2], see also [9, 4]. Moreover, the above quantity is equivalent to the total variation $|D f|\left((0,1)^{d}\right)$. In this case, the corresponding "vanishing subspace" is trivial:

$$
\lim _{\varepsilon \rightarrow 0} \varepsilon^{d-1} \sup _{\mathcal{G}_{\varepsilon}} \sum_{Q_{\varepsilon} \in \mathcal{G}_{\varepsilon}} \frac{1}{\left|Q_{\varepsilon}\right|} \int_{Q_{\varepsilon}}\left|f(x)-f_{Q_{\varepsilon}}\right| d x=0
$$

if and only if $f$ is constant.

Acknowledgments. We are grateful to the anonymous referee for several helpful suggestions. K.-M. Perfekt was partially supported by EPSRC grant EP/S029486/1. The research of R.Schiattarella has been funded by PRIN Project 2017JFFHSH.

L. D'Onofrio, L. Greco, C. Sbordone and R. Schiattarella are members of the Gruppo Nazionale per l'Analisi Matematica, la Probabilità e le loro Applicazioni (GNAMPA) of the Istituto Nazionale di Alta Matematica (INdAM).

\section{2. Атомic DECOMpositions}

We will suppose that $X$ is reflexive, while letting $Y$ be any Banach space. In this section, let $\left(L_{n}\right)_{n=1}^{\infty}$ be a given sequence of bounded operators $L_{n}: X \rightarrow Y$, and define

$$
E=\left\{x \in X: \sup _{n}\left\|L_{n} x\right\|_{Y}<\infty\right\} .
$$

We suppose that this defines a Banach space $E$ under the norm

$$
\|x\|_{E}=\sup _{n}\left\|L_{n} x\right\|_{Y}
$$

and that $E$ is continuously contained in $X$. We will not attempt to give a general condition under which these two hypotheses hold. We also suppose that $E$ is dense in $X$ in the $X$-norm, for otherwise we may replace $X$ by the closure of $E$ in $X$.

We thus have an isometric embedding

$$
V: E \rightarrow \ell^{\infty}(Y), \quad V x(n)=L_{n} x .
$$

The dual of $E$ can therefore be represented as

$$
E^{*} \simeq \mathrm{ba}\left(\mathbb{N}, Y^{*}\right) / V E^{\perp},
$$

where ba( $\left(\mathbb{N}, Y^{*}\right)$ denotes the space of finitely additive $Y^{*}$-valued set functions on $\mathbb{N}$ of bounded variation [12], and $V E^{\perp}$ is its subspace of elements annihilating $V E$. Note that $\ell^{1}\left(Y^{*}\right)$ is naturally understood as the subspace of $\mathrm{ba}\left(\mathbb{N}, Y^{*}\right)$ consisting of the countably additive measures.

Theorem 3. E has an isometric predual $E_{*}$,

$$
E_{*}=\ell^{1}\left(Y^{*}\right) / P,
$$


where $P=V E^{\perp} \cap \ell^{1}\left(Y^{*}\right)$. That is, every $x \in E$ corresponds to a functional on $\ell^{1}\left(Y^{*}\right) / P$ given by

$$
x\left(\left(y_{n}^{*}\right)\right)=\sum_{n=1}^{\infty} y_{n}^{*}\left(L_{n} x\right)=\sum_{n=1}^{\infty} L_{n}^{*} y_{n}^{*}(x), \quad\left(y_{n}^{*}\right) \in \ell^{1}\left(Y^{*}\right),
$$

and conversely every bounded functional on $\ell^{1}\left(Y^{*}\right) / P$ is given by a unique $x \in E$ according to (4). The norm of $x$ as a functional is equal to its norm as an element of $E$.

Proof. Through the canonical embedding $\iota: Y \rightarrow Y^{* *}$, we may instead consider the embedding

$$
W: E \rightarrow \ell^{\infty}\left(Y^{* *}\right), \quad W x(n)=\iota(V x(n)) .
$$

Note that $\ell^{\infty}\left(Y^{* *}\right)=\ell^{1}\left(Y^{*}\right)^{*}$ [12]. To see that $E$ is a dual space we simply have to verify that $W E$ is weak-star closed in $\ell^{\infty}\left(Y^{* *}\right)$. By the Krein-Smulian theorem, it is enough to check that $W E \cap D_{1}$ is weak-star closed, where $D_{1}$ is the closed unit ball with centre 0 in $\ell^{\infty}\left(Y^{* *}\right)$.

Suppose therefore that $\left(x_{\alpha}\right)$ is a net in the unit ball of $E$ such that $\left(L_{n} x_{\alpha}\right) \rightarrow\left(y_{n}^{* *}\right)$ weakstar, where $\left(y_{n}^{* *}\right) \in \ell^{\infty}\left(Y^{* *}\right)$. Since $E$ is continuously contained in $X$ and $X$ is reflexive, there is a subnet $\left(x_{\alpha^{\prime}}\right)$ such that $x_{\alpha^{\prime}} \rightarrow x$ weakly in $X$, for some $x \in X$. For every fixed $n$, $L_{n}: X \rightarrow Y$ is continuous, and thus $L_{n} x_{\alpha^{\prime}} \rightarrow L_{n} x$ weakly in $Y$. Since closed balls of $Y$ are weakly closed, we conclude that $x$ belongs to the unit ball of $E$. Of course, for every fixed $n$ we also know by hypothesis that $L_{n} x_{\alpha^{\prime}} \rightarrow y_{n}^{* *}$ weak-star in $Y^{* *}$. Then, for every $y^{*} \in Y^{*}$,

$$
y_{n}^{* *}\left(y^{*}\right)=\lim _{\alpha^{\prime}} y^{*}\left(L_{n} x_{\alpha^{\prime}}\right)=y^{*}\left(L_{n} x\right) .
$$

Hence $\left(y_{n}^{* *}\right)=\left(L_{n} x\right)$, demonstrating that $W E \cap D_{1}$ is weak-star closed.

We have shown that $E \simeq W E$ is a dual space, with predual given by

$$
(W E)_{*}=\ell^{1}\left(Y^{*}\right) /{ }^{\perp} W E=\ell^{1}\left(Y^{*}\right) / P .
$$

Remark. If $Y$ is a dual space with predual $Y_{*}$, a similar argument shows that

$$
E_{*}=\ell^{1}\left(Y_{*}\right){ }^{\perp} V E .
$$

To understand Theorem 3, note that finite sums $e_{*}=\sum L_{n}^{*} y_{n}^{*}$ belong both to $E_{*}$ and $X_{*}=X^{*}$, and the action of elements $x \in E$ on $e_{*}$ is identical,

$$
x\left(\left(y_{n}^{*}\right)\right)=e_{*}(x) .
$$

Clearly, such finite sums are dense in $E_{*}$. In fact, by restricting the action of $x^{*} \in X^{*}$ from $X$ to $E$, all of $X^{*}$ is continuously contained (and thus dense) in $E_{*}$.

Theorem 4. $X^{*}$ is continuously contained in $E_{*}$. Thus, for any $x^{*} \in X^{*}$ there is a sequence $\left(y_{n}^{*}\right) \in \ell^{1}\left(Y^{*}\right)$ such that $\left\|\left(y_{n}^{*}\right)\right\|_{\ell^{1}\left(Y^{*}\right)} \leq C\left\|x^{*}\right\|_{X^{*}}$ and

$$
x^{*}(x)=\sum_{n=1}^{\infty} L_{n}^{*} y_{n}^{*}(x), \quad x \in E .
$$

Here $C>0$ is an absolute constant. In particular, if $X$ is separable, then $E_{*}$ is separable.

Proof. Note first that it is clear that every $x^{*} \in X^{*}$ induces an element $x^{*} \in E^{*}$, since $E$ is continuously contained in $X$, and the implied map is injective. To see that in fact $x^{*} \in E_{*}$, we need to verify that $x^{*}$ is weak-star continuous on $E$. That is, we need to verify that $E \cap \operatorname{ker} x^{*}$ is weak-star closed in $E$. By the Krein-Smulian theorem, it is sufficient to consider $D_{1} \cap E \cap \operatorname{ker} x^{*}$, where $D_{1}$ is the closed unit ball with centre 0 in $E$. Let $\left(x_{\alpha}\right)$ be a net in this intersection such that $x_{\alpha} \rightarrow x$ weak-star in $E$. Since $E$ is continuously 
contained in $X$, there is a subnet $\left(x_{\alpha^{\prime}}\right)$ such that $x_{\alpha^{\prime}} \rightarrow x_{0}$ weakly in $X$ for some $x_{0} \in X$. By the same argument as in the proof of Theorem 3, we then have that $x_{0} \in D_{1} \cap E \cap \operatorname{ker} x^{*}$. Furthermore, for every $n$ and $y^{*} \in Y^{*}$, we find that

$$
y^{*}\left(L_{n} x_{0}\right)=\lim _{\alpha^{\prime}} y^{*}\left(L_{n} x_{\alpha^{\prime}}\right)=y^{*}\left(L_{n} x\right) .
$$

Hence $L_{n} x_{0}=L_{n} x$ for every $n$, and thus $x_{0}=x$ (by the assumption that the norm of $E$ is indeed a norm).

\section{BiduALITY AND DISTANCE FORMULAS}

In this section we will recall the framework and results of [13, 14], imposing additional structure on $E$. We now suppose that $X$ is separable, in addition to being reflexive. $Y$ is still an arbitrary Banach space. We assume that $E$ is normed by a collection $\mathcal{L}$ of operators $L: X \rightarrow Y$, equipped with a topology that is $\sigma$-compact, locally compact, Hausdorff, and such that $\mathcal{L} \ni L \rightarrow L x \in Y$ is continuous for every fixed $x \in X$. To reconcile this framework with that of Section 2, we additionally assume that the topology is separable.

Let

$$
E=\left\{x \in X: \sup _{L \in \mathcal{L}}\|L x\|_{Y}<\infty\right\}
$$

and

$$
E_{0}=\left\{x \in E: \varlimsup_{\mathcal{L} \ni L \rightarrow \infty}\|L x\|_{Y}=0\right\} .
$$

Here $L \rightarrow \infty$ is understood in the usual sense of escaping all compacts. As before, we assume that $E$ is a Banach space under the norm $\|x\|_{E}=\sup _{L \in \mathcal{L}}\|L x\|_{Y}$, and that $E$ is continuously contained and dense in $X$.

Additionally, we have to assume an approximation property.

(AP) For every $x \in E$ there is a sequence $\left(x_{n}\right) \subset E_{0}$ such that $\left\|x_{n}\right\|_{E} \leq\|x\|_{E}$ and $x_{n} \rightarrow x$ in $X$.

Since closed balls of $E_{0}$ may be viewed as bounded convex subsets of $X$, an equivalent reformulation of (AP) is obtained by replacing the strong convergence of $x_{n} \rightarrow x$ by weak convergence in $X$.

The following theorem was proven in $[13,14]$ by considering the natural embedding of $E$ into the space $C_{b}(\mathcal{L}, Y)$ of bounded continuous $Y$-valued functions on $\mathcal{L}$, and the corresponding embedding of $E_{0}$ into the space $C_{0}(\mathcal{L}, Y)$ of such functions vanishing at $\infty$. We refer the interested reader to [10] for the notion of an $M$-ideal. Let $\iota: E_{0} \rightarrow E_{0}^{* *}$ denote the canonical embedding.

Theorem 5 ([13, 14]). Suppose that (AP) holds. Then

- $E_{0}^{* *} \simeq E$ isometrically via the $X$ - $X^{*}$-pairing. More precisely, let $I: E_{0} \rightarrow X$ denote the inclusion operator, and let $U=I^{* *}$. Then $U\left(E_{0}^{* *}\right)=E$ and, considered as an operator $U: E_{0}^{* *} \rightarrow E, U$ is the unique isometric isomorphism such that $U \iota x=x$ for all $x \in E_{0}$.

- $E_{0}$ is an M-ideal in $E$.

- For every $x \in E$ it holds that

$$
\min _{x_{0} \in E_{0}}\left\|x-x_{0}\right\|_{E}=\varlimsup_{L \rightarrow \infty}\|L x\|_{Y}
$$


The uniqueness of $U$ has not been explicitly stated before, but follows as in [15, Theorem 2].

Choosing a dense sequence of operators $\left(L_{n}\right)$ in $\mathcal{L}$, we have by continuity that

$$
\|x\|_{E}=\sup _{n}\left\|L_{n} x\right\|_{Y}
$$

allowing us to apply the results of Section 2. Theorems 3 and 4 yield an isometric isomorphism $J: E \rightarrow E_{*}^{*}$ such that $J x\left(x^{*}\right)=x^{*}(x)$ for $x^{*} \in X^{*} \subset E_{*}$ and $x \in E$. Since $E_{0}$ is an $M$-ideal in $E_{0}^{* *}, E_{0}^{*}$ is a strongly unique predual, implying that the isometry $J U: E_{0}^{* *} \rightarrow E_{*}^{*}$ must arise as the adjoint $J U=K^{*}$ of an isometric isomorphism $K: E_{*} \rightarrow E_{0}^{*}$. Note, for $x^{*} \in X^{*} \subset E_{*}$ and $x \in E_{0}$, that

$$
K x^{*}(x)=J U \iota x\left(x^{*}\right)=x^{*}(x) .
$$

Theorem 6. Suppose that (AP) holds. Then the identity map, $K\left(x^{*}\right)=x^{*}, x^{*} \in X^{*}$, extends to an isometric isomorphism $K: E_{*} \rightarrow E_{0}^{*}$.

Less formally, we might simply say that $E_{0}^{*}=E_{*}$ and that $E_{*}^{*}=E$, isometrically via the $X-X^{*}$-pairing.

\section{The Bourgain-Brezis-Mironescu space}

We restrict the discussion to $d \geq 2$. If $d=1$, then $\mathcal{B}=$ BMO and the results are already known. If $d \geq 2$, [3, Theorem 2] states that $\mathcal{B}$ is continuously contained in the Marcinkiewicz space $L^{d /(d-1), \infty}$ (modulo constants). Therefore, as our choice of separable reflexive space containing $\mathcal{B}$, we may take $X=L^{p}\left((0,1)^{d}\right) / \mathbb{C}$, where $1<p<d /(d-1)$. Let $Y=L^{1}\left((0,1)^{d}\right)$.

As before, let $\mathcal{F}_{\varepsilon}$ denote a collection of disjoint open $\varepsilon$-cubes such that $\left|\mathcal{F}_{\varepsilon}\right| \leq \varepsilon^{1-d}$. Thus $\mathcal{F}_{\varepsilon}=\left(Q_{\varepsilon}\left(a_{j}\right)\right)_{j=1}^{m}$, where $Q_{\varepsilon}\left(a_{j}\right) \subset(0,1)^{d}$ is an $\varepsilon$-cube centered at $a_{j} \in(0,1)^{d}$, and $m \leq \varepsilon^{1-d}$. To each such collection $\mathcal{F}_{\varepsilon}$, associate the operator $L_{\mathcal{F}_{\varepsilon}}: L^{p} / \mathbb{C} \rightarrow L^{1}$ given by

$$
L_{\mathcal{F}_{\varepsilon}} f=\varepsilon^{d-1} \frac{1}{\left|Q_{\varepsilon}\right|} \sum_{j=1}^{\left|\mathcal{F}_{\varepsilon}\right|} \chi_{Q_{\varepsilon}\left(a_{j}\right)}\left(f-f_{Q_{\varepsilon}\left(a_{j}\right)}\right) .
$$

Since the cubes are disjoint, we then have that

$$
\left\|L_{\mathcal{F}_{\varepsilon}} f\right\|_{L^{1}}=\varepsilon^{d-1} \frac{1}{\left|Q_{\varepsilon}\right|} \sum_{j=1}^{\left|\mathcal{F}_{\varepsilon}\right|} \int_{Q_{\varepsilon}\left(a_{j}\right)}\left|f-f_{Q_{\varepsilon}\left(a_{j}\right)}\right| d x,
$$

and thus that

$$
\mathcal{B}=\left\{f \in L^{p} / \mathbb{C}: \sup _{0<\varepsilon \leq 1} \sup _{\mathcal{F}_{\varepsilon}}\left\|L_{\mathcal{F}_{\varepsilon}} f\right\|_{L^{1}}<\infty\right\} .
$$

To place ourselves in the framework of Section 3, we have to construct an appropriate topology on the set of collections $\mathcal{F}_{\varepsilon}$. Each collection is uniquely determined by $\varepsilon$ and the centres $a_{1}, \ldots, a_{m}$ of the cubes $(0,1)^{d} \supset Q_{\varepsilon}\left(a_{j}\right) \in \mathcal{F}_{\varepsilon}$. We thus write $\mathcal{F}_{\varepsilon}=\mathcal{F}\left(\varepsilon, a_{1}, \ldots, a_{m}\right)$. For each $m \in \mathbb{N}$, let

$$
\mathcal{L}_{m}=\left\{\mathcal{F}_{\varepsilon}=\mathcal{F}\left(\varepsilon, a_{1}, \ldots, a_{m}\right):\left|\mathcal{F}_{\varepsilon}\right|=m\right\}
$$

with the topology induced by the parametrization $\left(\varepsilon, a_{1}, \ldots, a_{m}\right) \in(0,1] \times(0,1)^{m d}$. Consider the map $\pi_{m}: \mathcal{L}_{m} \rightarrow\left(0, m^{-1 /(d-1)}\right]$ given by $\pi_{m}\left(\mathcal{F}_{\varepsilon}\right)=\varepsilon$. Since the cubes $Q_{\varepsilon}\left(a_{j}\right)$ are open, the map $\pi_{m}$ is proper. That is, $\pi_{m}^{-1}\left(\left[\delta, m^{-1 /(d-1)}\right]\right)$ is compact for every $0<\delta \leq$ $m^{-1 /(d-1)}$. By the dominated convergence theorem, the map $\mathcal{L}_{m} \ni \mathcal{F}_{\varepsilon} \mapsto L_{\mathcal{F}_{\varepsilon}} f \in L^{1}$ is continuous for every fixed $f \in L^{p}$ and $m$. 
We endow the full collection $\mathcal{L}=\left\{\mathcal{F}_{\varepsilon}\right\}$ with the disjoint union topology,

$$
\mathcal{L}=\coprod_{m=1}^{\infty} \mathcal{L}_{m}
$$

By the corresponding properties of $\mathcal{L}_{m}, \mathcal{L}$ is $\sigma$-compact, locally compact, Hausdorff, separable, and $\mathcal{L} \ni \mathcal{F}_{\varepsilon} \mapsto L_{\mathcal{F}_{\varepsilon}} f \in L^{1}$ is continuous for every $f \in L^{p}$. Furthermore, the map

$$
\pi=\coprod_{m=1}^{\infty} \pi_{m}: \mathcal{L} \rightarrow(0,1], \quad \pi\left(\mathcal{F}_{\varepsilon}\right)=\varepsilon,
$$

is proper, since $\pi_{m}$ is proper, and for every $\delta>0$ there is an $N$ such that $\pi^{-1}([\delta, 1]) \subset$ $\amalg_{m \leq N} \mathcal{L}_{m}$. That is, $\pi^{-1}([\varepsilon, 1])$ is compact for every $0<\varepsilon \leq 1$, and, by continuity, for every compact set $\mathcal{K} \subset \mathcal{L}$ there is an $\varepsilon>0$ such that $\mathcal{K} \subset \pi^{-1}([\varepsilon, 1])$. For a continuous function $F: \mathcal{L} \rightarrow \mathbb{R}$, we therefore have that

$$
\varlimsup_{\mathcal{L} \ni \mathcal{F}_{\varepsilon} \rightarrow \infty} F\left(\mathcal{F}_{\varepsilon}\right)=\varlimsup_{\varepsilon \rightarrow 0} \sup _{\mathcal{F}_{\varepsilon}} F\left(\mathcal{F}_{\varepsilon}\right) .
$$

Hence the Bourgain-Brezis-Mironescu space $\mathcal{B}$ and its vanishing counterpart $\mathcal{B}_{0}$ form an instance of the pair $\left(E, E_{0}\right)$, as described in Section 3,

$$
\mathcal{B}_{0}=\left\{f \in \mathcal{B}: \varlimsup_{\varepsilon \rightarrow 0} \sup _{\mathcal{F}_{\varepsilon}}\left\|L_{\mathcal{F}_{\varepsilon}} f\right\|_{L^{1}}=0\right\} .
$$

Before applying our results, we must prove that (AP) holds.

Lemma 7. For every $f \in \mathcal{B}$, there is a sequence $\left(f_{n}\right) \subset \mathcal{B}_{0}$ such that $\left\|f_{n}\right\|_{\mathcal{B}_{0}} \leq\|f\|_{\mathcal{B}}$ and $f_{n} \rightarrow f$ in $L^{p}, 1<p<d /(d-1)$.

Proof. For $0<\vartheta<\frac{1}{2}$, set $\boldsymbol{\vartheta}=(\vartheta, \ldots, \vartheta) \in \mathbb{R}^{d}$, and let

$$
g(x)=f((1-2 \vartheta) x+\boldsymbol{\vartheta}), \quad x \in(0,1)^{d} .
$$

Note that $g(x)$ is actually defined for all points $x \in(-\vartheta, 1+\vartheta)^{d}$. Choose a function $\psi \in$ $C_{c}\left(\mathbb{R}^{d}\right)$ such that $\psi \geq 0$, supp $\psi \subset(-1,1)^{d}$, and $\int \psi(y) \mathrm{d} y=1$. Fix $\vartheta$ and let $\varphi(y)=\varphi_{\vartheta}(y)=$ $\vartheta^{-d} \psi(y / \vartheta)$. Throughout the proof, integration with respect to $y$ will be understood to be taken over $\operatorname{supp} \varphi \subset(-\vartheta, \vartheta)^{d}$. Let

$$
h(x)=h_{\vartheta}(x)=\varphi * g(x)=\int \varphi(y) g(x-y) \mathrm{d} y .
$$

For cubes $Q_{\varepsilon} \subset(0,1)^{d}$, we use the notation

$$
M\left(f, Q_{\varepsilon}\right)=\frac{1}{\left|Q_{\varepsilon}\right|} \int_{Q_{\varepsilon}}\left|f-f_{Q_{\varepsilon}}\right| \mathrm{d} x .
$$

Note that

$$
\begin{aligned}
h(x)-h_{Q_{\varepsilon}} & =h(x)-\frac{1}{\left|Q_{\varepsilon}\right|} \int_{Q_{\varepsilon}}\left(\int \varphi(y) g(z-y) \mathrm{d} y\right) \mathrm{d} z \\
& =\int \varphi(y)\left(g(x-y)-g_{Q_{\varepsilon}-y}\right) \mathrm{d} y,
\end{aligned}
$$

and thus

$$
M\left(h, Q_{\varepsilon}\right) \leq \int \varphi(y) M\left(g, Q_{\varepsilon}-y\right) \mathrm{d} y .
$$


Furthermore,

$$
\begin{aligned}
M\left(g, Q_{\varepsilon}-y\right) & =\frac{1}{\left|Q_{\varepsilon}(y, \vartheta)\right|} \int_{Q_{\varepsilon}(y, \vartheta)}\left|f(u)-\frac{1}{\left|Q_{\varepsilon}(y, \vartheta)\right|} \int_{Q_{\varepsilon}(y, \vartheta)} f(v) \mathrm{d} v\right| \mathrm{d} u \\
& =M\left(f, Q_{\varepsilon}(y, \vartheta)\right)
\end{aligned}
$$

where $Q_{\varepsilon}(y, \vartheta)=\left\{(1-2 \vartheta) x+\boldsymbol{\vartheta}: x \in Q_{\varepsilon}-y\right\}$. Note that $Q_{\varepsilon}(y, \vartheta) \subset(0,1)^{d}$ is an $(1-2 \vartheta) \varepsilon_{-}$ cube, for every $y$.

Now consider a collection $\mathcal{F}_{\varepsilon}$ of disjoint $\varepsilon$-cubes such that $\left|\mathcal{F}_{\varepsilon}\right| \leq \varepsilon^{1-d}$. For each $y \in$ $\operatorname{supp} \varphi$

$$
\mathcal{F}_{\varepsilon}(y, \vartheta)=\left\{Q_{\varepsilon}(y, \vartheta): Q_{\varepsilon} \in \mathcal{F}_{\varepsilon}\right\}
$$

forms a collection of disjoint $(1-2 \vartheta) \varepsilon$-cubes, with

$$
\left|\mathcal{F}_{\varepsilon}(y, \vartheta)\right|=\left|\mathcal{F}_{\varepsilon}\right| \leq((1-2 \vartheta) \varepsilon)^{1-d} .
$$

Hence by (5),

$$
\begin{aligned}
\varepsilon^{d-1} \sum_{Q_{\varepsilon} \in \mathcal{F}_{\varepsilon}} M\left(h, Q_{\varepsilon}\right) & \leq \frac{1}{(1-2 \vartheta)^{d-1}} \int \varphi(y)((1-2 \vartheta) \varepsilon)^{d-1} \sum_{\mathcal{F}_{\varepsilon}(y, \vartheta)} M\left(f, Q_{\varepsilon}(y, \vartheta)\right) \mathrm{d} y \\
& \leq \frac{1}{(1-2 \vartheta)^{d-1}}\|f\|_{\mathcal{B}} .
\end{aligned}
$$

Therefore

$$
\|h\|_{\mathcal{B}} \leq \frac{1}{(1-2 \vartheta)^{d-1}}\|f\|_{\mathcal{B}} .
$$

To conclude, choose a sequence $\left(\vartheta_{n}\right)$ such that $\vartheta_{n} \rightarrow 0$, and let

$$
f_{n}=\left(1-2 \vartheta_{n}\right)^{d-1} h_{\vartheta_{n}} .
$$

Then $\left(f_{n}\right) \subset \mathcal{B}_{0},\left\|f_{n}\right\|_{\mathcal{B}_{0}} \leq\|f\|_{\mathcal{B}}$, and $f_{n} \rightarrow f$ in $L^{p}$.

Remark. Note that if $f \in \mathcal{B}_{0}$, then the sequence $\left(f_{n}\right)$ constructed in the proof of Lemma 7 converges to $f$ in $\mathcal{B}$. Hence the space UC of uniformly continuous functions on $(0,1)^{d}$ is dense in $\mathcal{B}_{0}$.

Indeed, the inequality (6) shows that

$$
\left[f_{n}\right]_{\varepsilon} \leq[f]_{(1-2 \vartheta) \varepsilon}, \quad 0<\varepsilon \leq 1 .
$$

Since $f \in \mathcal{B}_{0}$, there is for every $\sigma>0$ an $\varepsilon_{\sigma} \in(0,1)$ such that for $0<\varepsilon<\varepsilon_{\sigma}$ we have that

$$
[f]_{\varepsilon}<\sigma .
$$

Thus, for such $\varepsilon$,

$$
\left[f-f_{n}\right]_{\varepsilon} \leq[f]_{\varepsilon}+\left[f_{n}\right]_{\varepsilon}<2 \sigma .
$$

On the other hand, for any family $\mathcal{F}_{\varepsilon}$ of disjoint $\varepsilon$-cubes we have the trivial estimate

$$
\varepsilon^{d-1} \sum_{Q_{e} \in \mathcal{F}_{\varepsilon}} M\left(f-f_{n}, Q_{\varepsilon}\right) \leq 2 \varepsilon^{-1}\left\|f-f_{n}\right\|_{L^{1}\left((0,1)^{d}\right)} .
$$

Hence

$$
\lim _{n} \sup _{\varepsilon_{\sigma} \leq \varepsilon<1}\left[f-f_{n}\right]_{\varepsilon}=0
$$

since $f_{n} \rightarrow f$ in $L^{1}\left((0,1)^{d}\right)$. It follows that $f_{n} \rightarrow f$ in $\mathcal{B}$, since $\sigma$ was arbitrary. 
To prove Theorem 1, choose a dense sequence $\left(\mathcal{F}_{\varepsilon_{n}}^{n}\right) \subset \mathcal{L}$. Then Theorem 3 yields that for every $\varphi \in \mathcal{B}_{*}$ and $\delta>0$, there is a sequence $\left(y_{n}^{*}\right) \in \ell^{1}\left(L^{\infty}\left((0,1)^{d}\right)\right)$ such that $\sum\left\|y_{n}^{*}\right\|_{L^{\infty}}<\|\varphi\|_{\mathcal{B}_{*}}+\delta$ and

$$
\varphi=\sum_{n=1}^{\infty} L_{\mathcal{F}_{\varepsilon_{n}}^{n}}^{*} y_{n}^{*}=\sum_{n=1}^{\infty} L_{\mathcal{F}_{\varepsilon_{n}}^{n}} y_{n}^{*} .
$$

Then $\lambda_{n}=2\left\|y_{n}^{*}\right\|_{L^{\infty}}$ and $g_{n}=L_{\mathcal{F}_{\varepsilon_{n}}^{n}} y_{n}^{*} /\left(2\left\|y_{n}^{*}\right\|_{L^{\infty}}\right)$ satisfy the desired properties, $\varphi=\sum_{n} \lambda_{n} g_{n}$, and

$$
\sum_{n=1}^{\infty}\left|\lambda_{n}\right|<2\|\varphi\|_{\mathcal{B}_{*}}+2 \delta
$$

Conversely, suppose that we are given a functional $\varphi=\sum_{n} \lambda_{n} g_{n}$ of the form in the theorem. Let $\mathcal{F}_{\varepsilon_{n}}^{n}$ denote the collection of cubes associated with $g_{n}$. If necessary we may complete $\left(\mathcal{F}_{\varepsilon_{n}}^{n}\right)$ to a dense sequence. Theorem 3 then shows that $\varphi$ is weak-star continuous on $\mathcal{B}$, and it is immediate that

$$
\|\varphi\|_{\mathcal{B}_{*}} \leq \sum_{n=1}^{\infty}\left|\lambda_{n}\right|
$$

Remark. As Theorem 3 shows, one obtains an isometric description of $\mathcal{B}_{*}$ by considering atoms of the form $\tilde{g}_{n}=L_{\mathcal{F}_{\varepsilon_{n}}^{n}} y_{n}^{*}$, normalized by the fact that $\left\|y_{n}^{*}\right\|_{L^{\infty}}=1$.

Theorem 2 follows directly from Theorems 5 and 6 .

\section{REFERENCES}

[1] Ambrosio, Luigi, Bourgain, Jean, Brezis, Haïm and Figalli, Alessio. BMO-type norms related to the perimeter of sets. Comm. Pure Appl. Math., 69 (2016), 1062-1086.

[2] Ambrosio, Luigi and Comi, Giovanni. Anisotropic Surface Measures as Limits of Volume Fractions. Current Research in Nonlinear Analysis 135 (2018), 1-32.

[3] Bourgain, Jean, Brezis, Haïm and Mironescu, Petru. A new function space and applications. J. Eur. Math. Soc. (JEMS) 17 (2015), no. 9, 2083-2101.

[4] Brudnyi, Alexander and Brudnyi, Yuri. On Banach Structure of Multivariate BV Spaces I. (2018), arXiv:1806.08824.

[5] D’Onofrio, Luigi, Sbordone, Carlo and Schiattarella, Roberta. Duality and distance formulas in Banach function spaces. J. Elliptic Parabol. Equ. (2018).

[6] Dafni, Galia, Hytönen, Tuomas, Korte, Riikka and Yue, Hong. The space JNp: nontriviality and duality. J. Funct. Anal. 275 (2018), no. 3, 577-603.

[7] Farroni, Fernando, Fusco, Nicola, Guarino Lo Bianco, Serena and Schiattarella, Roberta. A formula for the anisotropic total variation of SBV functions, to appear on J. Funct. Anal.

[8] Fusco, Nicola, Moscariello, Gioconda and Sbordone, Carlo. A formula for the total variation of SBV functions. J. Funct. Anal. 270 (2016), no. 1, 419-446.

[9] Fusco, Nicola, Moscariello, Gioconda and Sbordone, Carlo. BMO-type seminorms and Sobolev functions. ESAIM Control Optim. Calc. Var. 24 (2018), no. 2, 835-847.

[10] Harmand, Peter, Werner, Dirk, and Werner, Wend. $M$-ideals in Banach spaces and Banach algebras. Lecture Notes in Mathematics, 1547. Springer-Verlag, Berlin, 1993. viii+387 pp.

[11] Holmes, Richard, Scranton, Bruce, and Ward, Joseph. Approximation from the space of compact operators and other $M$-ideals. Duke Math. J. 42 (1975), 259-269.

[12] Leonard, Isaac E. Banach sequence spaces. J. Math. Anal. Appl. 54 (1976), no. 1, 245-265.

[13] Perfekt, Karl-Mikael. Duality and distance formulas in spaces defined by means of oscillation. Ark. Mat. 51 (2013), no. 2, 345-361.

[14] Perfekt, Karl-Mikael. On M-ideals and o-O type spaces. Math. Scand. 121 (2017), no. 1, 151-160.

[15] Perfekt, Karl-Mikael. On the spaces of bounded and compact multiplicative Hankel operators. New York J. Math. 25 (2019), 589-602. 
Dipartimento di Scienze e Tecnologie, Università degli Studi di Napoli "Parthenope", Centro Direzionale Isola C4, 80100 Napoli, ITALY

Email address: donofrio@uniparthenope.it

Dipartimento di Ingegneria Elettrica e delle Tecnologie dell' Informazione, Università degli Studi di Napoli "Federico II", Via Claudio 21, 80125 Napoli, Italy

Email address: luigreco@unina.it

Department of Mathematics and Statistics, University of Reading, Reading RG6 6AX, United Kingdom Email address: k.perfekt@areading.ac.uk

Dipartimento di Matematica e Applicazioni "R. Caccioppoli", Università degli Studi di Napoli "Federico II", Via Cintia, 80126 Napoli, Italy

Email address: sbordone@unina.it

Dipartimento di Matematica e Applicazioni "R. Caccioppoli", Università degli Studi di Napoli "Federico II", Via Cintia, 80126 Napoli, Italy

Email address: roberta.schiattarella@unina.it 